\title{
Optical polarization of gamma-ray bright blazars
}

\author{
Dmitry Blinov ${ }^{1}$, E. Angelakis, M. Balokovic, L. Fuhrmann, \\ T. Hovatta, K. Katarzyski, P. Khodade, O. King, A. Kus, N. Kylafis, \\ I. Myserlis, G. Panopoulou, I. Papadakis, I. Papamastorakis, \\ V. Pavlidou, B. Pazderska, E. Pazderski, T. Pearson, C. Rajarshi, \\ A. Ramaprakash, A. Readhead, P. Reig, R. Rouneq, K. Tassis, and \\ A. Zensus \\ ${ }^{1}$ University of Crete, Greece \\ E-mail: dmitriy.blinov@gmail.com
}

\begin{abstract}
We report about first results of the RoboPol project. RoboPol is a large-sample, high-cadence, polarimetric monitoring program of blazars in optical wavelengths, using a camera specifically constructed for this project, mounted at the University of Crete's Skinakas Observatory $1.3 \mathrm{~m}$ telescope. The analysis of RoboPol data is conducted in conjunction with Fermi LAT gamma-ray data, and multifrequency radio data from the OVRO (Caltech), FGAMMA (MPIfR), and Torun (NCU) monitoring programs. Using carefully selected samples of gamma-ray bright and weak blazars we investigate a connection between their optical polarization behaviour and variability properties in gamma. We examine a relationship of gamma flares with polarization angle rotations relying on robust statistical criteria. We analyse also the optical polarization variability itself in order to establish some restrictions on physical models of blazars jets.
\end{abstract}

